\title{
Estudos de repetitividade e reprodutividade para dados funcionais
}

\author{
Alexandre Homsi Pedotta*, Flávio Sanson Fogliatto ${ }^{\mathrm{b}}$ \\ a*pedott@producao.ufrgs.br, UFRGS, Brasil \\ bffogliatto@producao.ufrgs.br, UFRGS, Brasil
}

\begin{abstract}
Resumo
Este artigo apresenta um método adaptado do estudo de repetitividade e reprodutividade (R\&R) para analisar a capacidade e o desempenho de sistemas de medição, no contexto da análise de dados funcionais. Dados funcionais ocorrem quando a variável de resposta é caracterizada por uma coleção de dados que formam um perfil ou uma curva, e não por uma observação individual. 0 método proposto é uma alternativa ao uso de métodos tradicionais de análise, que são ineficientes para dados funcionais. 0 método apresenta uma adaptação da análise de variância de dois fatores. Foi usada a distância de Hausdorff como medida de proximidade entre as curvas. 0 método proposto foi aplicado a um estudo simulado de R\&R. 0 estudo foi estruturado para analisar cenários em que o sistema de medição foi aprovado e reprovado. Os resultados foram comparados com os obtidos através da aplicação da ANOVA ponto a ponto nas curvas. 0 método proposto foi denominado de ANOVA das distâncias.
\end{abstract}

Palavras-chave

Análise de dados funcionais. Sistemas de medição. Estudos de R\&R. ANOVA. ANOVA funcional.

\section{Introdução}

A análise de dados funcionais (ADF) configura-se como necessidade crescente para a Engenharia da Qualidade (EQ). A ausência de técnicas adaptadas para dados funcionais leva à utilização de métodos equivocados na otimização de produtos e processos por eles caracterizados. 0 emprego de métodos desenvolvidos para variáveis de respostas com valor único pode deteriorar a confiabilidade de um produto ou processo cuja otimização envolva respostas funcionais (FOGLIATTO, 2008).

A avaliação do desempenho e da capacidade de um sistema de medição (SM) envolve a análise de diferentes tipos de características de qualidade (CQs). Existem CQs para as quais o resultado da amostragem da variável de interesse é representado por uma coleção de dados que pertencem a um perfil ou uma curva. A partir desses pontos é possível estimar os parâmetros que descrevem a curva. 0 conteúdo informativo de uma curva é maior do que o de um ponto individual. Os valores discretos medidos são pontos de uma função dependente de outra variável, denominada indexadora. Ramsay e Silverman (2005) denominam coleções de dados dependentes de variáveis indexadoras de dados funcionais. A função que representa o conjunto de dados pode ser obtida através de um processo de interpolação, utilizando técnicas de suavização.

0 estudo de repetitividade \& reprodutividade (R\&R) é uma ferramenta da EQ usada na análise de SMs. Nos estudos de R\&R, o instrumento de medição é usado para medir repetidas vezes as amostras de um produto. A repetitividade se refere à variabilidade característica do instrumento de medição, e decorre da sua capacidade de fornecer leituras repetidas muito próximas, sob as mesmas condições. A reprodutibilidade se refere à capacidade de um SM apresentar os mesmos resultados a partir da alteração nas condições de medição, tais como mudanças de avaliadores, diferentes turnos de trabalho, ou alterações de processo. 0 objetivo do estudo de R\&R é determinar se a variabilidade do SM é relativamente menor que a variabilidade do processo monitorado (INTERNATIONAL..., 2008; BURDICK; BORROR; MONTGOMERY, 2003; AUTOMOTIVE..., 2002). 
0 método de análise mais usado em estudos de R\&R é a análise de variância (ANOVA - Analysis of Variance). A ANOVA permite decompor a variabilidade do SM e avaliar a interação entre suas componentes. A estimativa da variabilidade do sistema é obtida a partir da variância do erro aleatório, dado pelas replicações. 0 erro de medição é composto pela dispersão do instrumento, pelo efeito do avaliador e pelo erro aleatório devido às replicações. Os critérios mais usados para avaliar a capacidade de um SM são: (i) índice de capacidade de medição $\left(I C M_{1}\right)$, dado como um percentual de variabilidade do processo; (ii) índice de capacidade de medição $\left(I C M_{2}\right)$, dado como um percentual da variabilidade da especificação do processo; e (iii) razão sinal-ruído $(S N R)$ ou número de categorias distintas $(n d c)$. 0 ICM indica a distorção da variação do processo devida ao SM. O $I C M_{2}$ representa a habilidade do instrumento de classificar os produtos frente às especificações. $0 n d c$ reflete a capacidade do SM de discriminar categorias, dentro da variação do processo. A indústria automotiva, por exemplo, recomenda o uso do $I C M_{1}$ e o $n d c$ nas avaliações dos sistemas de medições (BARRENTINE, 2003; BURDICK; BORROR; MONTGOMERY, 2005; AUTOMOTIVE..., 2002).

A maior parte das pesquisas recentes que envolvem estudos de R\&R enfoca a análise de SMs, cuja variável de resposta é dada por um valor individual. Majeske (2008) apresenta um método de análise para SMs com múltiplas variáveis de respostas independentes. 0 método foi aplicado a um SM formado por um dispositivo que mede diferentes CQs, simultaneamente. 0 método envolve a aplicação da ANOVA multivariada (MANOVA) e apresenta critérios de aceitação para os indicadores $I C M_{1}, I C M_{2}$ e $n d c$. Tal método, entretanto, não pode ser usado no caso de dados funcionais.

Pesquisas recentes em ADF focalizam a representação dos dados, a determinação de comportamentos padronizados e as variações entre diferentes curvas. Ferraty e Vieu (2003) empregam o estimador de Kernel para classificar curvas a categorias existentes, em algo que pode ser visto como uma análise discriminante funcional. Ramsay e Silverman (2005) apresentam as adaptações de diferentes técnicas multivariadas, tais como análise de componentes principais e modelos lineares de suavização por Splines, para o contexto funcional. Abramovich e Angelini (2006) apresentam um modelo de análise de variância para dados funcionais (FANOVA - Functional Analysis of Variance). 0 modelo estabelece um procedimento para teste de significância baseado nos coeficientes de wavelet empíricos, que permite caracterizar diferentes tipos de respostas funcionais suavizadas sob alternativas não paramétricas. Tal análise funcional ancorada em testes estatísticos sobre os coeficientes de modelos ajustados às curvas está em consonância com a proposta de Kang e Albin (2000), trabalho precursor no tratamento de dados funcionais no controle estatístico da qualidade.

0 controle estatístico da qualidade de dados funcionais é denominado de monitoramento de perfil (MP). 0 MP é um campo de pesquisa relativamente novo, mas que cresce rapidamente. 0 MP é usado para avaliar a estabilidade das curvas ao longo do tempo. Entre as principais demandas estão a capacidade de detectar qualquer alteração na forma da função e a simplicidade do modelo do perfil (WOODALL, 2007).

Outra adaptação para a análise de variância de dados funcionais foi proposta por Cuevas, Febrero e Fraiman (2004). Trata-se de uma abordagem funcional pura, não havendo necessidade de discretizar os pontos das curvas para a análise dos dados. A proposta está baseada no comportamento assintótico do teste $F$ clássico da ANOVA. A expressão para o cálculo de $F$ apresenta no numerador a variabilidade das curvas entre os grupos e no denominador a variabilidade das curvas dentro dos grupos. 0 método usa um teste baseado no numerador do teste $F$.

O objetivo deste trabalho é adaptar a ANOVA para avaliar um SM em que variáveis funcionais são mensuradas. Para tanto, utiliza-se uma abordagem baseada no cálculo de distâncias entre curvas. Através do uso dessas distâncias, variáveis funcionais são reduzidas a variáveis simples. A ANOVA das distâncias é então testada em duas abordagens. As abordagens se diferenciam pela forma de cálculo da distância entre as curvas. Dentre as medidas de distância entre curvas disponíveis na literatura, optou-se pelo uso da distância de Hausdorff, dada a sua generalidade (HUTTENLOCHER; KLANDERMAN; RUCKLIDGE, 1993).

0 método proposto contribui de forma significativa para o aumento da eficiência de estudos de R\&R, onde a variável de resposta é funcional. A adaptação baseada em distâncias proposta para a ANOVA na ADF representa uma evolução sobre a abordagem tradicional, devida a Ramsay e Silverman (2005). Nela, as curvas sob análise são discretizadas em pontos ancorados na variável indexadora (por exemplo, o tempo no qual a observação foi coletada) e a análise é desdobrada em ANOVAs individuais, cada uma incidindo sobre pontos coincidentes nas curvas (por exemplo, pontos nas diferentes curvas observados no mesmo instante de tempo). A menos que as ANOVAs individuais apresentem o mesmo resultado, não será possível concluir sobre a existência ou não de diferença significativa entre as curvas. $\mathrm{Na}$ abordagem aqui proposta um único resultado final será obtido e a conclusão sobre o desempenho do SM decorrerá diretamente desse resultado. 
A seção seguinte apresenta uma revisão sobre o teste ANOVA para dois fatores e sua aplicação em estudos de R\&R. Na seção 3 é apresentada a ANOVA das distâncias, desenvolvida para a análise de estudos de $R \& R$ para variáveis funcionais. Na seção 4 são apresentados os resultados da aplicação do método proposto a dados simulados baseados em um estudo de caso em uma indústria de fabricação de pneus. A discussão dos resultados e as conclusões são apresentadas na seção 5 .

\section{Abordagem clássica da ANOVA em um estudo de R\&R}

Um experimento clássico para o estudo de R\&R usa a ANOVA de dois fatores. 0 primeiro fator é a peça (P) e o outro, o avaliador (A). 0 modelo estatístico para a variável de resposta é dado pela Equação 1. 0 modelo clássico considera fatores com efeitos fixos. 0 efeito é considerado fixo quando os níveis incluem todas as possibilidades do fator; isto é, todos os avaliadores que operam o sistema são incluídos e cada avaliador avalia as mesmas peças padronizadas (MONTGOMERY; RUNGER, 2003).

$$
x_{i j k}=\mu+\tau_{i}+\beta_{j}+(\tau \beta)_{i j}+\epsilon_{i j k}\left\{\begin{array}{l}
i=1, \ldots, l \\
j=1, \ldots, J \\
k=1, \ldots, k
\end{array}\right.
$$

Na Equação 1, $x_{i j k}$ é o valor da variável de resposta para a observação da $k$-ésima repetição feita pelo $i$-ésimo avaliador sobre a $j$-ésima peça, $\mu$ é a é o valor da média geral, $\tau_{i}$ é o efeito do $i$-ésimo avaliador, $\beta_{j}$ é o efeito da $j$-ésima peça, $(\tau \beta)_{i j}$ é o efeito da interação entre o avaliador $i$ e a peça $j$, e $\varepsilon_{i j k}$ é o resíduo ou erro aleatório, normalmente distribuído e com média zero. 0 resíduo é dado pela diferença entre os valores observados e esperados para a variável de resposta, conforme mostra o modelo matemático da Equação 2, onde $x_{i j k}$ é o valor observado e $\bar{x}_{i j}$ representa o valor médio da variável de resposta da $j$-ésima peça medida pelo $i$-ésimo avaliador. A ANOVA pressupõe observações normalmente e independentemente distribuídas, com idêntica variância nos diferentes níveis dos fatores. Segundo Montgomery (2001) e Montgomery e Runger (2003), desvios moderados da normalidade não implicam em uma violação séria do pressuposto.

$\varepsilon_{i j k}=x_{i j k}-\bar{x}_{i j}$.

O objetivo da ANOVA é testar a hipótese de igualdade das médias ou dos efeitos dos fatores e da interação serem iguais a zero. As variâncias devem ser calculadas a partir das somas quadradas dos resíduos. A soma quadrática total (SQT) é dada na Equação 3. A soma quadrática dos avaliadores (SQA) é dada na Equação 4. A soma quadrática das peças (SQP) é dada na Equação 5. A soma quadrática da interação entre os avaliadores e as peças (SQAP) é dada na Equação 6. Por fim, a soma quadrática dos resíduos $(S Q R)$ é dada na Equação 7.

$S Q T=\sum_{i=1}^{l} \sum_{j=1}^{J} \sum_{k=1}^{K}\left(x_{i j k}-\bar{x}_{. . .}\right)^{2}$

$S Q A=J K \sum_{i=1}^{l}\left(x_{i . .}-\bar{x}_{\ldots}\right)^{2}$

$S Q P=I K \sum_{j=1}^{J}\left(x_{. j .}-\bar{x}_{\ldots .}\right)^{2}$

$S Q A P=K \sum_{i=1}^{l} \sum_{j=1}^{J}\left(\bar{x}_{i j .}-\bar{x}_{i . .}-\bar{x}_{. j .}+\bar{x}_{\ldots . .}\right)^{2}$

$S Q R=\sum_{i=1}^{l} \sum_{j=1}^{J} \sum_{k=1}^{K}\left(x_{i j k}-\bar{x}_{i j}\right)^{2}$

A identidade que relaciona a SQT às demais somas quadráticas é dada por:

$S Q T=S Q A+S Q P+S Q A P+S Q R$,

com cada termo apresentando o seguinte número de graus de liberdade (GDL):

$$
\begin{aligned}
& (I J K-1)=(I-1)+(J-1)+(I-1) \\
& (J-1)+I J(K-1)
\end{aligned}
$$

As médias quadráticas de cada fator são dadas pela razão entre as somas quadradas e os GDL dos respectivos fatores. Se o efeito de um fator for significativo, o valor esperado da média quadrática do fator deve ser diferente do valor esperado da média quadrática dos resíduos. A verificação da existência de diferença significativa entre o valor esperado para a média quadrática do fator e a média quadrática dos resíduos é feita pela aplicação do teste $F$. 0 teste $F$ é aplicado para cada fator e interação do estudo. Em geral, a interação não deve ser significativa. Neste caso, é recomendável unificar as somas quadradas. A unificação consiste em somar SQAPà SQR; da mesma forma, deve-se somar os graus de liberdade para a nova formulação. A adição das somas quadradas e o novo número de graus de liberdade geram novos valores para as médias quadráticas e correspondentes valores calculados de $F$ (MONTGOMERY; RUNGER, 2003).

AIAG (AUTOMOTIVE..., 2002) apresenta o cálculo da decomposição da variabilidade de um 
SM. A repetitividade ou variação do equipamento é denominada $V E$. A reprodutividade ou variação dos avaliadores é denominada $V A$. A variação do SM é avaliada pelo cálculo do $R \& R$. A variação total do estudo é denominada de $V T$. VPé a variação da peça ou processo. A variabilidade de cada fator é comparada à variabilidade total do SM. A Tabela 1 apresenta 0 cálculo da percentagem que cada fator contribui para a variação total do sistema. As componentes da variação do SM são representadas pelo desvio padrão 5,15 sigma. 0 desvio padrão é usado porque é mais fácil interpretá-lo do que a variância. No caso da $M Q A P$ ser maior que a $M Q A$ a $V A$ deve ser considerada nula. No caso da $M Q A P$ ser maior que a $M Q P$ a $V P$ deve ser considerada nula. No caso da $M Q R$ ser maior que a $M Q A P$ a $V A P$ deve ser considerada nula (AUTOMOTIVE..., 2002).

Os indicadores de capacidade dos sistemas de medição são calculados da seguinte forma:

$I C M_{1}=\% R \& R$

$n d c=\sqrt{2} \times \frac{V P}{R \& R}$
A Tabela 2 apresenta critérios de decisão recomendados para avaliação de um SM (BARRENTINE, 2003; BURDICK; BORROR; MONTGOMERY, 2005; AUTOMOTIVE..., 2002).

\section{ANOVA das distâncias}

Considere um estudo clássico de R\&R, conforme apresentado na seção 2 . 0 problema consiste em avaliar o desempenho de um SM cuja característica de qualidade é uma variável funcional. Na abordagem clássica a variável de resposta é medida por um valor único, dado pela Equação 1. Na ADF, a variável de resposta é medida e o resultado é um conjunto de pontos, que pertencem a um perfil ou uma curva. A cada ponto desse conjunto existe um valor associado de uma variável indexadora, usualmente o tempo no qual o valor da variável de resposta foi observado. 0 conjunto de valores medidos associado à variável indexadora pode ser organizado em um vetor.

Sejam $l$ avaliadores e $J$ peças diferentes. Cada avaliador efetua $K$ repetições da medição de uma CQ cuja variável de resposta é funcional. A variável de resposta é formada por conjuntos de $N$ pontos, pertencentes a um perfil ou curva. As observações da

Tabela 1. Componentes da variabilidade de um SM.

\begin{tabular}{lcc}
\hline \multicolumn{1}{c}{ Fonte de variação } & Desvio padrão 5,15 sigma & Percentual da variação total \\
\hline Repetitividade & $V E=5,15 \times \sqrt{M Q R}$ & $\% V E=100 \times \frac{V E}{V T}$ \\
Reprodutibilidade & $V A=5,15 \times \sqrt{\frac{M Q A-M Q A P}{l K}}$ & $\% V A=100 \times \frac{V A}{V T}$ \\
Interação P $\mathrm{A}$ & $V A P=5,15 \times \sqrt{\frac{M Q A P-M Q R}{K}}$ & $\% V A P=100 \times \frac{V A P}{V T}$ \\
Peça & $V P=5,15 \times \sqrt{\frac{M Q P-M Q A P}{J K}}$ & $\% V P=100 \times \frac{V P}{V T}$ \\
R\&R & $R \& R=\sqrt{(V E)^{2}+(V A)^{2}+(V A P)^{2}}$ & $\% R \& R=100 \times \frac{R \& R}{V T}$ \\
Total & $V T=\sqrt{(R \& R)^{2}+(V P)^{2}}$ & \\
\hline
\end{tabular}

Tabela 2. Critérios de aceitação para a capacidade de sistemas de medição.

\begin{tabular}{lll}
\hline \multicolumn{1}{c}{ Critérios } & \multicolumn{1}{c}{ Decisão } & \multicolumn{1}{c}{ Observação } \\
\hline$l C M_{1} \leq 0,1$ & $\mathrm{SM}$ aprovado & Depende da capacidade do processo e dos custos de seleção do produto \\
$0,1<l C M_{1} \leq 0,3$ & $\mathrm{SM}$ pode ser aprovado & \\
$l C M_{1}>0,3$ & $\mathrm{SM}$ reprovado & Depende da capacidade do processo e dos custos de seleção do produto \\
$n d c \leq 2$ & $\mathrm{SM}$ reprovado & \\
$2<n d c<5$ & $\mathrm{SM}$ pode ser aprovado & \\
$n d c \geq 5$ & $\mathrm{SM}$ aprovado & \\
\hline
\end{tabular}


$k$-ésima repetição do $i$-ésimo avaliador sobre a $j$-ésima peça estão organizadas no vetor $x_{i j k}$, no espaço $\mathbb{R}^{2}$.

$\mathrm{x}_{i j k}=\left[\left(x_{i j k}, t_{1}\right), \ldots,\left(x_{i j k N}, t_{N}\right)\right]$

onde $i=1, \ldots, l, j=1, \ldots, J, k=1, \ldots, K$ e $n=1, \ldots, N$ são inteiros e positivos, e t é um número real não negativo correspondendo ao valor da variável indexadora.

Considerando que a variável de resposta é uma curva, os resíduos usados nos cálculos da variância na ANOVA devem ser determinados através de uma medida de proximidade entre curvas; uma medida possível é dada pela distância entre elas. A distância entre duas curvas pode ser calculada através da distância entre pontos representativos das curvas. Fogliatto (2008) usou a distância de Hausdorff (DH) para converter respostas funcionais em respostas simples em um contexto de otimização de experimentos com múltiplas respostas. A DH fornece uma medida da distância entre duas curvas ou conjuntos de pontos diferentes. Quanto menor a distância, maior a semelhança entre os dois conjuntos. A DH é definida como o limite superior (valor máximo) do conjunto das distâncias mínimas entre os pontos de dois vetores. A definição de $\mathrm{DH}$ pode variar de acordo com os objetivos; por exemplo, o operador de máximo pode ser substituído pela mediana, ou pela soma das distâncias mínimas. Souza (2008) usou diferentes medidas de distâncias para testar se a curva média de um grupo de curvas é igual a uma curva previamente conhecida.

A ANOVA das distâncias aqui proposta se baseia no cálculo da DH entre curvas observadas e curvas médias. Uma curva média é definida pelo conjunto das médias dos pontos das curvas observadas.

Seja $\overline{\mathrm{x}}_{i . .}$ o vetor da média do avaliador $i$ dado por:

$\overline{\mathbf{x}}_{i . .}=\left[\left(\frac{\sum_{j=1}^{J} \sum_{k=1}^{K} x_{i j k 1}}{J K}, t_{1}\right), \ldots,\left(\frac{\sum_{j=1}^{J} \sum_{k=1}^{K} x_{i j k N}}{J K}, t_{N}\right)\right]$

Seja $\overline{\mathbf{x}}_{. j .}$ o vetor da média da peça $j$ dado por:

$\overline{\mathbf{x}}_{. j .}=\left[\left(\frac{\sum_{i=1}^{l} \sum_{k=1}^{K} x_{i j k 1}}{I K}, t_{1}\right), \ldots,\left(\frac{\sum_{i=1}^{l} \sum_{k=1}^{K} x_{i j k N}}{I K}, t_{N}\right)\right]$

Seja $\overline{\mathrm{x}}_{i j}$. o vetor da média do avaliador $i$ e da peça $j$, dado por:

$\overline{\mathbf{x}}_{i j .}=\left[\left(\frac{\sum_{k=1}^{K} x_{i j k 1}}{K}, t_{1}\right), \ldots,\left(\frac{\sum_{k=1}^{K} x_{i j k N}}{K}, t_{N}\right)\right]$

Seja $\bar{x} \ldots$ o vetor da média geral, correspondente a todas as observações, dado por:

$\overline{\mathrm{x}}_{\mathrm{..}}=\left[\left(\frac{\sum_{i=1}^{l} \sum_{j=1}^{J} \sum_{k=1}^{K} x_{i j k 1}}{l J K}, t_{1}\right), \ldots .,\left(\frac{\sum_{i=1}^{l} \sum_{j=1}^{J} \sum_{k=1}^{K} x_{i j k N}}{l J K}, t_{N}\right)\right]$

As distâncias entre as curvas observadas [Equação 12] e as curvas médias das Equações 13 a 16 estão associadas às variabilidades características do SM. A ANOVA das distâncias será apresentada em duas abordagens. As abordagens se diferenciam pelo cálculo da distância entre as curvas. A primeira abordagem calcula a DH através da mediana. A segunda calcula a DH através da média. Por ser uma estatística robusta, o uso da mediana resulta em uma abordagem mais conservadora, se comparada à da média.

\subsection{Abordagem das medianas}

A partir da Equação 3 obtém-se a soma quadrática total (SQT) dada por:

$S Q T=\sum_{i=1}^{l} \sum_{j=1}^{J} \sum_{k=1}^{K}\left[d_{x i j k}\left(\bar{x}_{\ldots}\right)\right]^{2}$

onde $d_{x i j k}\left(\bar{x}_{\ldots}\right)$, obtida pela Equação 18 , é a $\mathrm{DH}$ entre cada curva observada $x_{i j k}$ em um determinado 
grupo e a curva da média geral $\bar{x}_{\text {... }}$. Essa distância é equivalente ao resíduo entre o valor observado e o valor da média geral $\left(x_{i j k}-\bar{x}_{\ldots}\right)$.

$d_{\mathrm{x} j \mathrm{k} k}\left(\overline{\mathrm{x}}_{\ldots}\right)=$ mediana $_{\bar{x}_{\ldots, \ldots} \in \overline{\mathrm{x}} \ldots . .} d\left(\bar{x}_{\ldots, .}, \mathrm{x}_{i j k}\right)$

onde,

$d\left(\bar{x}_{\ldots n}, x_{i j k}\right)=\min _{x_{i j k} \in x_{i j k}} d\left(\bar{x}_{\ldots n}, x_{i j k}\right)$

sendo a distância $d\left(\bar{x}_{\ldots .}, x_{i j k}\right)$ correspondente à distância euclidiana entre um ponto do vetor $\overline{\mathbf{x}}_{\text {... }}$ e um ponto do vetor $\mathbf{x}_{i j k}$; considerando-se os pontos $\bar{X}_{. .1}$ e $x_{111}$, essa distância seria dada por:

$d\left(\bar{x}_{\ldots 1}-x_{111}\right)=\sqrt{\left(\bar{x}_{\ldots 1}-x_{111}\right)^{2}+\left(t_{\bar{x}_{\ldots 1}}-t_{x_{111}}\right)^{2}}$

A Equação 20 mostra que a distância euclidiana está na base do cálculo da DH. Esse cálculo, entretanto, faz com que se perca a informação da posição da curva observada em relação à curva média. Isto é, duas curvas diferentes podem ter a mesma $\mathrm{DH}$, porém estarem em posições simétricas em relação à curva média. A alternativa para não perder essa informação é atribuir um sinal a cada distância calculada. 0 sinal será positivo se o valor da resposta do ponto for maior que o valor da curva média, no mesmo instante $t$, e negativo se menor. A informação do sinal deve ser usada para a verificação dos pressupostos de normalidade dos resíduos. Os resíduos são dados pelas distâncias entre as curvas observadas $x_{i j k}$ e as curvas médias $\overline{\mathbf{x}}_{i j}$. Para uma melhor exatidão do teste $F$ da ANOVA os valores dos resíduos devem apresentar distribuição normal e variância constante. Desvios moderados da suposição de normalidade não afetam significativamente o resultado do teste.

A Figura 1 apresenta um desenho esquemático das distâncias definidas pelas Equações 18 e 19. As retas tracejadas representam as distâncias de um ponto da curva média para cada ponto de uma curva individual. A menor distância foi representada pela reta de linha sólida. Um valor mínimo de distância deve ser associado a cada ponto da curva média. Esses dados formam o conjunto de $N$ distâncias usadas no cálculo da mediana.

A soma quadrática dos avaliadores (SQA) é obtida a partir da Equação 4, sendo dada por:

$$
S Q A=J K \sum_{i=1}^{l}\left[d_{\bar{x}_{i . .}}\left(\bar{x}_{. . .}\right)\right]^{2}
$$

onde $d_{\bar{x}_{i . .}}\left(\bar{x}_{\ldots}\right)$ é a distância entre a curva média $\overline{\mathrm{X}}_{i . .}$ de cada avaliador e a curva da média geral $\overline{\mathbf{x}}_{\text {... }}$, dada por:

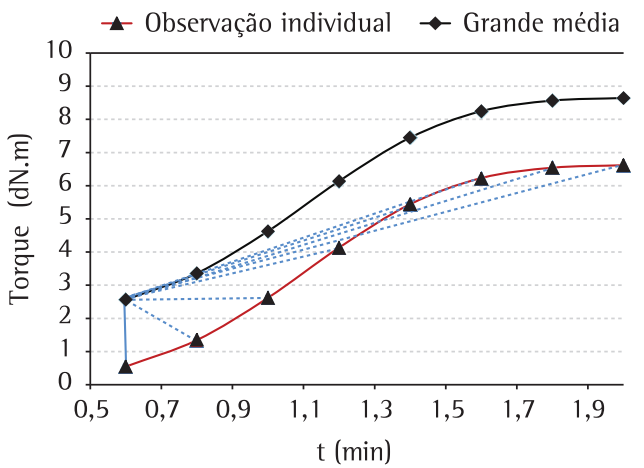

Figura 1. Definição da DH.

$d_{\overline{\mathrm{x}}_{i . .}}\left(\overline{\mathrm{x}}_{\ldots}\right)=$ mediana $_{X_{\ldots . . n} \in \overline{\mathrm{x}}_{\ldots} . . .} d\left(\bar{x}_{\ldots n}, \overline{\mathrm{x}}_{i}\right)$

onde,

$d\left(\bar{x}_{\ldots n}, \overline{\mathbf{x}}_{i . .}\right)=\min _{\bar{x}_{i . .} \in \bar{x}_{i . .}} d\left(\bar{x}_{\ldots n}, \bar{x}_{i . .}\right)$

sendo a distância $d\left(\bar{x}_{\ldots n}, \bar{x}_{i . .}\right)$ definida na Equação 20.

Da Equação 5 se obtém a soma quadrática das peças (SQP), dada por:

$S Q P=I K \sum_{j=1}^{J}\left[d_{\bar{x}_{. j .}}\left(\bar{x}_{\ldots}\right)\right]^{2}$

onde $d_{\overline{\mathrm{x}}_{. j .}}\left(\overline{\mathrm{x}}_{\ldots}\right)$ é a distância entre a curva média $\overline{\mathrm{x}}_{. j}$. de cada peça e a curva da média geral $\overline{\mathrm{x}}_{\text {... }}$, dada por:

$d_{\overline{\mathrm{x}}_{. j .} .}\left(\overline{\mathrm{x}}_{\ldots}\right)=$ mediana $_{\chi_{\ldots . . n} \in \overline{\mathrm{x}}_{\mathrm{x}} . .} d\left(x_{\ldots n}, \overline{\mathrm{x}}_{. j .}\right)$

onde,

$d\left(\bar{x}_{\ldots n}, \overline{\mathrm{x}}_{. j .}\right)=\min _{\bar{x}_{. j .} \in \bar{x}_{. j .}} d\left(\bar{x}_{\ldots n}, \bar{x}_{. j .}\right)$

sendo a distância $d\left(\bar{x}_{\ldots n}, \bar{x}_{. j .}\right)$ definida na Equação 20.

A soma quadrática para a interação entre os avaliadores e as peças (SQAP) corresponde à diferença das distâncias dadas pelas Equações 25 e 28, sendo dada por:

$$
\begin{aligned}
& S Q A P=K \sum_{i=1}^{l} \sum_{j=1}^{J}\left(\bar{x}_{i j .}-\bar{x}_{i . .}-\bar{x}_{. j .}+\bar{x}_{. . .}\right)^{2}= \\
& =K \sum_{i=1}^{l} \sum_{j=1}^{J}\left(\left(\bar{x}_{i j .}-\bar{x}_{i . .}\right)-\left(\bar{x}_{. j .}+\bar{x}_{\ldots . .}\right)\right)^{2}= \\
& =S Q A P=K \sum_{i=1}^{l} \sum_{j=1}^{J}\left(d_{\bar{x}_{i j} .}\left(\bar{x}_{i . .}\right)-d_{\bar{x}_{. j .} .}\left(\bar{x}_{\ldots}\right)\right)
\end{aligned}
$$

onde $d_{\bar{x}_{i j} .}\left(\overline{\mathrm{x}}_{i . .}\right)$ é a distância entre a curva média $\overline{\mathrm{x}}_{i j}$. das $K$ repetições e a curva média $\overline{\mathbf{x}}_{i .}$ do respectivo avaliador $i$, dada por: 
$d_{\bar{x}_{i j .}}\left(\bar{x}_{i . .}\right)=\operatorname{mediana}_{\bar{x}_{i . . n} \in \overline{\mathrm{X}}_{i}} d\left(\bar{x}_{i . . n}, \bar{x}_{i j .}\right)$

onde,

$d\left(\bar{x}_{i . . n}, \bar{x}_{i j .}\right)=\min _{\bar{x}_{\bar{x} j} \in \bar{x}_{j j .}} d\left(\bar{x}_{i . . n}, \bar{x}_{i j .}\right)$

sendo a distância $d\left(\bar{x}_{i . . n}, \bar{x}_{i j}\right)$ definida pela Equação 20.

Por fim, a soma quadrática dos resíduos $(S Q R)$ é obtida a partir da Equação 7, sendo dada por:

$$
S Q R=\sum_{i=1}^{l} \sum_{j=1}^{J} \sum_{k=1}^{K}\left[d_{x_{i j k}}\left(\bar{x}_{i j} .\right)\right]^{2}
$$

onde $d_{\mathrm{x}_{i j k}}\left(\overline{\mathrm{x}}_{i j .}\right)$ é a distância entre cada curva observada $\mathbf{x}_{i j k}$ e a curva média $\overline{\mathbf{x}}_{i j}$. dos respectivos avaliador $i$ e peça $j$, dada por:

$d_{x_{i j k}}\left(\bar{x}_{i j .}\right)=\operatorname{mediana}_{\bar{x}_{i j .} \in \bar{x}_{i j} .} d\left(\bar{x}_{i j . n}, \mathrm{x}_{i j k}\right)$

onde,

$d\left(\bar{x}_{i j . n}, \mathrm{x}_{i j k}\right)=\min _{x_{i j k} \in \mathrm{x}_{i j k}} d\left(\bar{x}_{i j . n}, x_{i j k}\right)$

sendo a distância $d\left(\bar{x}_{i j . n}, x_{i j k}\right)$ definida pela Equação 20.

Existem duas alternativas para o cálculo de $S Q R$. A primeira alternativa é usar diretamente a Equação 30. A segunda é usar a identidade associando a Equação 8 às Equações 17, 21, 24 e 27. As duas alternativas podem levar a resultados diferentes. A diferença ocorre porque o valor esperado usado na Equação 30 pode variar de curva para curva. Por exemplo, se a DH da observação $x_{111}$ estiver associada ao tempo $t=t_{1}$ $(n=1)$ da curva média $\overline{\mathbf{X}}_{11}$ e a DH da observação $\mathrm{x}_{112}$ estiver associada ao tempo $\mathrm{t}=\mathrm{t}_{2}(n=2)$ da curva média $\overline{\mathbf{X}}_{11}$, os valores esperados de $\bar{x}_{11 . n}$ usados no cálculo da Equação 32 serão diferentes; isto é, $\bar{X}_{11.1} \neq \bar{X}_{11.2}$. Essa diferença depende da variação ao longo de $t$.

A escolha da primeira alternativa pode levar a pequenos desvios na identidade da Equação 8. A escolha da segunda alternativa pode levar a uma pequena distorção no valor de $S Q R$. A escolha da fórmula de cálculo do método deve levar em conta o impacto nos erros tipo 1 e 11. 0 número de GDL deve ser calculado de acordo com a Equação 9. As médias quadráticas são dadas pela Equações 34 a 37.

$$
M Q A=\frac{J K \sum_{i=1}^{l}\left[d_{\bar{x}_{i . .}}\left(\bar{x}_{\ldots}\right)\right]^{2}}{(l-1)}
$$

$M Q P=\frac{I K \sum_{j=1}^{J}\left[d_{\bar{x}_{. j .} .}\left(\bar{x}_{\ldots}\right)\right]^{2}}{(J-1)}$

$$
M Q A P=\frac{K \sum_{i=1}^{l} \sum_{j=1}^{J}\left(d_{\overline{\mathrm{x}}_{i j .}}\left(\overline{\mathrm{x}}_{i . .}\right)-d_{\overline{\mathrm{x}}_{. j .}}\left(\overline{\mathrm{x}}_{\ldots}\right)\right)}{(l-1)(J-1)}
$$

$$
M Q R=\frac{\sum_{i=1}^{l} \sum_{j=1}^{J} \sum_{k=1}^{K}\left[d_{x_{i j k}}\left(\bar{x}_{i j}\right)\right]^{2}}{I J(K-1)}
$$

Se o efeito de um fator for significativo, o valor esperado da média quadrática do fator deve ser diferente do valor esperado da média quadrática dos resíduos. A tabela da ANOVA para o modelo da Equação 1 é apresentada na Tabela 3. 0 teste $F$ determina se os efeitos do avaliador, da peça e da interação são significativos. Se o valor calculado da estatística $F\left(F_{\text {CAL }}\right)$ for maior que o valor tabelado de $F$, deve-se rejeitar a hipótese nula. Caso o efeito da interação não seja significativo, a tabela da ANOVA deve ser reformulada para unificar a variância da interação à variância dos resíduos.

A Tabela 3 fornece as informações suficientes para avaliar o desempenho do SM. A avaliação é baseada no cálculo das percentagens que cada componente contribui para a variação total do SM. Tais percentagens são calculadas conforme apresentado na Tabela 1. A capacidade do SM deve ser avaliada pelos indicadores $I C M_{1}$ e o $n d c$, descritos nas Equações 10 e 11. Os critérios de aprovação devem ser os mesmos apresentados na Tabela 2.

\subsection{Abordagem da média}

A distância dada pela mediana pode não apontar valores extremos em pontos específicos das curvas, o que poderia levar a uma interpretação equivocada dos resultados. Uma alternativa seria substituir o operador de mediana usado nas Equações 18, 22, 25, 28 e 31 pelo operador de média. Para diferenciar as distâncias calculadas nas duas abordagens, denota-se a distância calculada através da média por $d_{x_{i j k}}^{a}\left(\bar{x}_{\ldots}\right)$. 0 cálculo das somas quadráticas segue o mesmo procedimento da abordagem da mediana. A SQT, por exemplo, é dada pela Equação 37. Com isso, a tabela ANOVA para essa abordagem é similar àquela apresentada na Tabela 3. Os critérios de aprovação devem ser os mesmos apresentados na Tabela 2.

$$
S Q T=\sum_{i=1}^{l} \sum_{j=1}^{J} \sum_{k=1}^{K} \sum_{n=1}^{N}\left[d_{x_{i j k}}^{a}\left(\bar{x}_{\text {... }}\right)\right]^{2}
$$




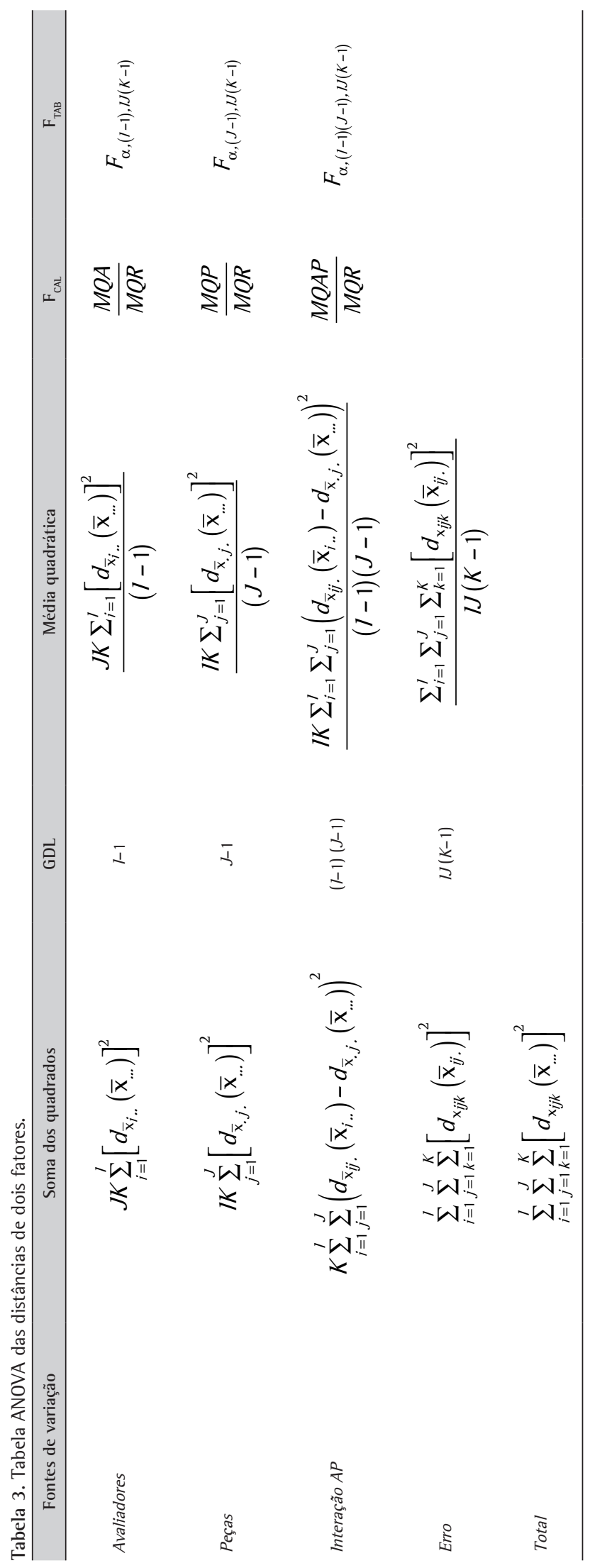


onde $d_{\mathrm{x}_{i j k}}^{a}\left(\overline{\mathrm{x}}_{\ldots}\right)$ é a DH entre cada curva observada $\mathbf{x}_{i j k}$ em um determinado grupo e a curva da grande média $\overline{\mathbf{x}}_{\text {... }}$, dada pela Equação 38.

$d_{\mathrm{x}_{i j k}}^{a}\left(\overline{\mathrm{x}}_{\ldots}\right)=$ média $_{x_{\ldots . . n} \in \overline{\mathrm{x}} . . .} d\left(x_{\ldots n}, \mathrm{x}_{i j k}\right)$

onde,

$d\left(x_{\ldots . . .}, \mathrm{x}_{i j k}\right)=\min _{x_{i j k} \in \mathrm{x}_{i j k}} d\left(x_{\ldots . . n}, x_{i j k}\right)$

sendo a distância $d\left(x_{\ldots . n}, x_{i j k}\right)$ definida pela Equação 20.

\section{Aplicação prática}

Esta seção apresenta o resultado da aplicação dos métodos propostos na seção 3 a um estudo de R\&R. 0 estudo foi baseado no caso de um fabricante de pneus, que avalia a qualidade dos produtos através da viscosidade da borracha. A medição é feita em um reômetro de laboratório. 0 reômetro é um instrumento que permite medir as propriedades viscoelásticas da borracha. 0 instrumento fornece a curva reométrica de vulcanização da borracha como resultado. A partir da curva reométrica são obtidos os valores usuais para caracterização da borracha. Uma característica viscosa é dada pelo torque suportado por uma amostra de borracha em função do tempo. As medidas de torque são tomadas em diferentes instantes do tempo de vulcanização.

Com base em dados reais do fabricante de pneus, verificou-se que a curva de vulcanização da borracha segue o modelo de Weibull, apresentado na Equação 40. Os dados foram ajustados através de modelos de regressão não linear.

$$
x=\beta_{0}-\beta_{1} e^{-\beta_{2} \beta^{\beta_{3}}}
$$

Nessa equação, $X$ representa o torque, $\beta_{o}, \beta_{1,} \beta$ e $\beta_{3}$ são os coeficientes experimentais do modelo e t o tempo em minutos. A partir desse modelo, é possivel determinar o valor do torque em qualquer tempo no intervalo modelado, o que permite que a curva de vulcanização seja comparável em intervalos fixos de tempo.

0 objetivo da simulação aqui apresentada foi gerar curvas correspondentes às $K$ medições dos $l$ avaliadores efetuadas sobre as $J$ peças de referência. Foram gerados dados para a obtenção de quatro cenários no contexto da avaliação de um SM. No primeiro cenário foram geradas curvas tal que o estudo de R\&R resultasse na aprovação do SM. No segundo cenário foram simuladas curvas que resultaram na reprovação do SM. Neste caso, o SM foi reprovado devido ao efeito do avaliador. No terceiro cenário foram geradas curvas que resultaram na reprovação do SM, devido ao efeito do equipamento de medição. Nesses três cenários iniciais, o resultado da avaliação do SM utilizando a ANOVA das distâncias ou a ANOVA ponto a ponto é concordante. No quarto cenário simulado foram geradas curvas que novamente resultaram na reprovação do SM devido ao efeito do equipamento de medição, mas onde os resultados da ANOVA das distâncias e da ANOVA ponto a ponto divergem. As duas abordagens propostas na seção 3 foram usadas na análise de cada cenário simulado. Os resultados foram sempre comparados com aqueles obtidos utilizando a ANOVA ponto a ponto nas curvas, seguindo a proposta de Ramsay e Silverman (2005).

0 estudo simulado de R\&R envolve a avaliação de um SM com 2 avaliadores e 5 peças. Cada avaliador efetua 5 repetições da medida, cuja variável de resposta é funcional. A variável de resposta é formada por curvas compostas de 11 pontos. A primeira fase do processo de simulação foi a obtenção de 5 curvas de referência correspondentes às 5 peças de referência. As curvas de referência foram obtidas a partir do modelo da Equação 40. A Tabela 4 apresenta os parâmetros de ajuste $\beta_{0}, \beta_{1}, \beta_{2}$ e $\beta_{3}$ de cada curva de referência usados para o estudo simulado. A Figura 2 apresenta a forma das curvas. As curvas não representam, necessariamente, a variação do processo.

A segunda fase do processo de simulação foi a geração das curvas observadas para cada cenário.

Tabela 4. Parâmetros de ajuste das curvas de referência.

\begin{tabular}{cccccc}
\hline Parâmetro & Peça 1 & Peça 2 & Peça 3 & Peça 4 & Peça 5 \\
\hline$\beta_{0}$ & 6,672 & 6,722 & 6,772 & 6,822 & 6,872 \\
$\beta_{1}$ & 6,263 & 6,263 & 6,263 & 6,263 & 6,263 \\
$\beta_{2}$ & 0,159 & 0,159 & 0,159 & 0,159 & 0,159 \\
$\beta_{3}$ & 2,936 & 2,936 & 2,936 & 2,936 & 2,936 \\
\hline
\end{tabular}

Cunvas de referência
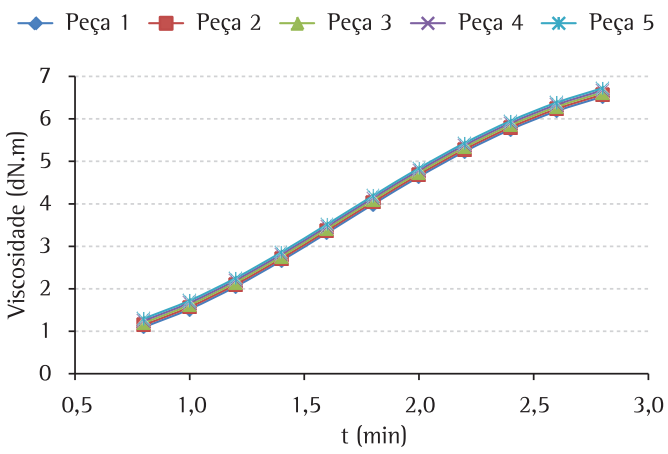

Figura 2. Curvas das 5 peças de referência. 
Tabela 5. Parâmetros das distribuições dos erros aleatórios.

\begin{tabular}{llcccc}
\hline & & SM Aprovado & SM Reprovado VA & SM Reprovado VE' & SM Reprovado VE $^{2}$ \\
\hline \multirow{2}{*}{ Erro avaliador 1 } & $\mu=$ & 0 & 0 & 0 & 0 \\
& $\sigma=$ & 0,002 & 0,002 & 0,002 & 0,02 \\
Erro avaliador 2 & $\mu=$ & 0 & 0,05 & 0 & 0 \\
& $\sigma=$ & 0,002 & 0,002 & 0,002 & 0,02 \\
Erro SM 1 & $\mu=$ & 0 & 0 & 0 & 0 \\
& $\sigma=$ & 0,002 & 0,002 & 0 & 0 \\
Erro SM 2 & $\mu=$ & 0 & 0,02 & 0,1 \\
Erro SM 3 & $\sigma=$ & 0,002 & 0 & 0 & 0 \\
& $\mu=$ & 0 & 0,002 & 0,1 & 0,002 \\
\hline
\end{tabular}

Para simular os valores medidos, foram adicionados dois termos de erro aleatório à Equação 40. Foram usados erros aleatórios seguindo uma distribuição normal com parâmetros $\mu$ e $\sigma 2$. 0 primeiro termo de erro corresponde à influência do avaliador sobre o SM; o segundo corresponde ao erro característico do equipamento de medição. 0 erro do equipamento de medição foi dividido em três fatores, devido a diferenças de escala dentro do perfil que caracteriza a variável de resposta funcional. A Tabela 5 apresenta os parâmetros das distribuições dos termos de erro usados em cada cenário. 0 erro SM1 corresponde ao erro do SM para os valores de $t$ menores que 1,6 minuto; o erro SM2 corresponde ao erro do SM para os valores de t entre 1,6 e 2,2 minutos; o erro SM3 corresponde ao erro do SM para valores de t maiores que 2,2 minutos.

Em todos os cenários, o teste Kolmogorov-Smirnov de normalidade não rejeitou a hipótese de normalidade dos dados, a um nível de confiança de $\alpha=0,05$. Da mesma forma, em todos os cenários simulados o efeito da interação não foi significativo; os termos de $S Q A P$ foram assim unificados aos da $S Q R$.

\subsection{SM aprovado}

A Tabela 6 apresenta os resultados dos testes de hipóteses que compõem a ANOVA utilizando a abordagem das medianas, na seção 3.1, das médias, na seção 3.2, e da ANOVA ponto a ponto; os resultados nos três casos foram muito similares. 0 teste $F$ mostra que há efeito significativo para a variação das peças nos três casos. 0 efeito dos avaliadores não foi significativo. Foi usada a Equação 30 para o cálculo da $S Q R$.

Na segunda coluna da Tabela 6 , tem-se o resultado dos estudos de R\&R (valores em percentual do indicador $I C M_{1}$ da Tabela 2, para as três abordagens. Os valores apresentados aprovam o SM com folga nos três casos, já que todos estão abaixo do valor limite de 10\%. Também não há conflito de resultados
Tabela 6. Resultados da ANOVA de dois fatores para o cenário simulado.

\begin{tabular}{|c|c|c|c|c|c|c|}
\hline \multicolumn{2}{|c|}{ Abordagem } & $\% R \& R$ & $\mathrm{H}_{0} \mathrm{~A}$ & $\mathrm{H}_{0} \mathrm{P}$ & $\mathrm{H}_{0} \mathrm{AP}$ & $\neq S Q T$ \\
\hline \multicolumn{2}{|l|}{ Mediana } & 1,3146 & & Rejeita & & 0,0000 \\
\hline \multicolumn{2}{|l|}{ Média } & 1,0957 & & Rejeita & & 0,0000 \\
\hline \multirow{11}{*}{$\begin{array}{l}\text { Ponto a } \\
\text { ponto }\end{array}$} & 0,8 & 3,7767 & & Rejeita & & 0,0000 \\
\hline & 1,0 & 3,5183 & & Rejeita & & 0,0000 \\
\hline & 1,2 & 3,4249 & & Rejeita & & 0,0000 \\
\hline & 1,4 & 4,2191 & & Rejeita & & 0,0000 \\
\hline & 1,6 & 3,3582 & & Rejeita & & 0,0000 \\
\hline & 1,8 & 3,7747 & & Rejeita & & 0,0000 \\
\hline & 2,0 & 3,5882 & & Rejeita & & 0,0000 \\
\hline & 2,2 & 3,3575 & & Rejeita & & 0,0000 \\
\hline & 2,4 & 3,0845 & & Rejeita & & 0,0000 \\
\hline & 2,6 & 2,7952 & & Rejeita & & 0,0000 \\
\hline & 2,8 & 3,6353 & & Rejeita & & 0,0000 \\
\hline
\end{tabular}

na ANOVA ponto a ponto (que inicia em $t=0,8$ e termina em $t=2,8$ ). As três colunas seguintes informam os resultados dos testes de hipótese que compõem a ANOVA de dois fatores. $\mathrm{H}_{0} \mathrm{~A}, \mathrm{H}_{0} \mathrm{P}$, e $\mathrm{H}_{0}$ $\mathrm{AP}$ correspondem à hipótese nula da não existência de efeito do avaliador, da peça e da interação entre avaliador e peça, respectivamente. Somente a rejeição de $\mathrm{H}_{0}$ é informada na tabela, a um nível de confiança de $\alpha=0,05$. A última coluna da Tabela 6 traz o desvio na identidade da Equação 8.

A Tabela 7 apresenta os indicadores de capacidade e desempenho do SM no cenário aprovado, para as duas primeiras abordagens.

0 resultado da Tabela 7 é compatível com o cenário simulado. 0 SM foi aprovado nas duas abordagens. 0 desvio na identidade da Equação 8 não se fez notar até a quinta casa após a vírgula. Considerando os critérios de aprovação da Tabela 2, o SM foi aprovado.

\subsection{SM reprovado devido à $V A$}

A Tabela 8 apresenta os resultados dos testes de hipóteses que compõem a ANOVA utilizando a 
abordagem das medianas, das médias e da ANOVA ponto a ponto. É possível observar a grande similaridade nos resultados das três abordagens. 0 teste $F$ permite concluir pela existência de efeito significativo para a variação das peças e dos avaliadores nas três abordagens.

A Tabela 9 apresenta os indicadores de capacidade e desempenho do SM no cenário de reprovação devido à VA, para as duas primeiras abordagens.

0 resultado da Tabela 9 é compatível com o cenário simulado. O SM foi reprovado nas três abordagens. A fonte de variação que mais contribuiu para a reprovação foi a VA. 0 desvio na identidade da Equação 8 apresentou valores maiores, se comparados aos da Tabela 6. Considerando os critérios de aprovação da Tabela 2, o SM foi reprovado.

\subsection{SM reprovado devido à $V E$}

A Tabela 10 apresenta os resultados dos testes de hipóteses que compõem a ANOVA utilizando a abordagem das medianas, das médias e da ANOVA

Tabela 7. Comparação dos percentuais de variação do SM para o cenário simulado.

\begin{tabular}{ccc}
\hline Fonte de variação & Mediana & Média \\
\hline \%VE & 1,3 & 1,1 \\
\%VA & 0,0 & 0,0 \\
\%VP & 100,0 & 100,0 \\
$\% R \& R$ & 1,3 & 1,1 \\
$n d c$ & 107,57 & 129,06 \\
\hline
\end{tabular}

Tabela 8. Resultados da ANOVA de dois fatores para o cenário simulado.

\begin{tabular}{|c|c|c|c|c|c|c|}
\hline \multicolumn{2}{|c|}{ Abordagem } & \%R\&R & $\mathrm{H}_{0} \mathrm{~A}$ & $\mathrm{H}_{0} \mathrm{P}$ & $\mathrm{H}_{0} \mathrm{AP}$ & $\neq S Q T$ \\
\hline \multicolumn{2}{|l|}{ Mediana } & 41,1925 & Rejeita & Rejeita & & $-0,0006$ \\
\hline \multicolumn{2}{|l|}{ Média } & 41,1931 & Rejeita & Rejeita & & $-0,0003$ \\
\hline \multirow{11}{*}{$\begin{array}{l}\text { Ponto a } \\
\text { ponto }\end{array}$} & 0,8 & 41,9439 & Rejeita & Rejeita & & 0,0000 \\
\hline & 1,0 & 41,4054 & Rejeita & Rejeita & & 0,0000 \\
\hline & 1,2 & 41,6762 & Rejeita & Rejeita & & 0,0000 \\
\hline & 1,4 & 41,5020 & Rejeita & Rejeita & & 0,0000 \\
\hline & 1,6 & 40,8099 & Rejeita & Rejeita & & 0,0000 \\
\hline & 1,8 & 41,5717 & Rejeita & Rejeita & & 0,0000 \\
\hline & 2,0 & 40,6352 & Rejeita & Rejeita & & 0,0000 \\
\hline & 2,2 & 41,2623 & Rejeita & Rejeita & & 0,0000 \\
\hline & 2,4 & 40,2708 & Rejeita & Rejeita & & 0,0000 \\
\hline & 2,6 & 40,5071 & Rejeita & Rejeita & & 0,0000 \\
\hline & 2,8 & 41,3126 & Rejeita & Rejeita & & 0,0000 \\
\hline
\end{tabular}

Tabela 9. Comparação dos percentuais de variação do SM para o cenário simulado.

\begin{tabular}{ccc}
\hline Fonte de variação & Mediana & Média \\
\hline$\% \mathrm{VE}$ & 1,3 & 1,1 \\
$\% \mathrm{VA}$ & 41,2 & 41,1 \\
$\% \mathrm{VP}$ & 91,1 & 91,2 \\
$\% \mathrm{R} \& \mathrm{R}$ & 41,2 & 41,2 \\
$n d c$ & 3,13 & 3,14 \\
\hline
\end{tabular}

ponto a ponto. As duas primeiras abordagens apresentam valores similares de $\% R \& R$, os quais divergem daqueles obtidos na ANOVA ponto a ponto. Nas três abordagens reprova-se o SM, com o teste $F$ mostrando efeito significativo para a variação das peças.

A Tabela 11 apresenta os indicadores de capacidade e desempenho do SM no cenário de reprovação devida à VE, para as duas primeiras abordagens.

0 resultado da Tabela 11 é compatível com o cenário simulado. 0 SM foi reprovado nas três abordagens. A fonte de variação que mais contribuiu para a reprovação foi a VE. 0 desvio na identidade da Equação 8 apresentou valores maiores do que aqueles da Tabela 8. Considerando os critérios de aprovação da Tabela 2, o SM foi reprovado.

\subsection{SM reprovado devido à VE e com resultados inconclusivos na ANOVA ponto a ponto}

A Tabela 12 apresenta os resultados dos testes de hipóteses que compõem a ANOVA utilizando a abordagem das medianas, das médias e da ANOVA ponto a ponto. As três abordagens apresentam valores distintos de $\% R \& R$, os quais podem ser explicados analisando o gráfico das curvas resultantes da simulação na Figura 3. As curvas plotadas são as médias sobre todas as replicações obtidas em cada grupo de avaliador e peça, além das duas curvas

Tabela 10. Resultados da ANOVA de dois fatores para o cenário simulado.

\begin{tabular}{|c|c|c|c|c|c|c|}
\hline \multicolumn{2}{|c|}{ Abordagem } & $\% R \& R$ & $\mathrm{H}_{0} \mathrm{~A}$ & $\mathrm{H}_{0} \mathrm{P}$ & $\mathrm{H}_{0} \mathrm{AP}$ & $\neq S Q T$ \\
\hline \multicolumn{2}{|l|}{ Mediana } & 40,9653 & & Rejeita & & 0,0541 \\
\hline \multicolumn{2}{|l|}{ Média } & 35,5959 & & Rejeita & & $-0,0122$ \\
\hline \multirow{11}{*}{$\begin{array}{l}\text { Ponto a } \\
\text { ponto }\end{array}$} & 0,8 & 76,1036 & & Rejeita & & 0,0000 \\
\hline & 1,0 & 80,4017 & & Rejeita & & 0,0000 \\
\hline & 1,2 & 85,0410 & & Rejeita & & 0,0000 \\
\hline & 1,4 & 87,1818 & & Rejeita & & 0,0000 \\
\hline & 1,6 & 83,6490 & & Rejeita & & 0,0000 \\
\hline & 1,8 & 57,6463 & & Rejeita & & 0,0000 \\
\hline & 2,0 & 86,4875 & & Rejeita & & 0,0000 \\
\hline & 2,2 & 83,6940 & & Rejeita & & 0,0000 \\
\hline & 2,4 & 78,1121 & & Rejeita & & 0,0000 \\
\hline & 2,6 & 78,7864 & & Rejeita & & 0,0000 \\
\hline & 2,8 & 82,5900 & & Rejeita & & 0,0000 \\
\hline
\end{tabular}

Tabela 11. Comparação dos percentuais de variação do SM para o cenário simulado.

\begin{tabular}{ccc}
\hline Fonte de variação & Mediana & Média \\
\hline \%VE & 41,0 & 35,6 \\
\%VA & 0,0 & 0,0 \\
\%VP & 91,2 & 93,5 \\
\%R\&R & 41,0 & 35,6 \\
$n d c$ & 3,15 & 3,71 \\
\hline
\end{tabular}


Tabela 12. Resultados da ANOVA de dois fatores para o cenário simulado.

\begin{tabular}{|c|c|c|c|c|c|c|}
\hline \multicolumn{2}{|c|}{ Abordagem } & \%R\&R & $\mathrm{H}_{0} \mathrm{~A}$ & $\mathrm{H}_{0} \mathrm{P}$ & $\mathrm{H}_{0} \mathrm{AP}$ & $\neq S Q T$ \\
\hline \multicolumn{2}{|l|}{ Mediana } & 2,3483 & & Rejeita & & 0,0006 \\
\hline \multicolumn{2}{|l|}{ Média } & 22,9935 & & Rejeita & & $-0,0027$ \\
\hline \multirow{11}{*}{$\begin{array}{l}\text { Ponto a } \\
\text { ponto }\end{array}$} & 0,8 & 2,8606 & & Rejeita & & 0,0000 \\
\hline & 1,0 & 3,7235 & & Rejeita & & 0,0000 \\
\hline & 1,2 & 4,0454 & & Rejeita & & 0,0000 \\
\hline & 1,4 & 3,8809 & & Rejeita & & 0,0000 \\
\hline & 1,6 & 69,6413 & & Rejeita & & 0,0000 \\
\hline & 1,8 & 84,3594 & & Rejeita & & 0,0000 \\
\hline & 2,0 & 82,2729 & & Rejeita & & 0,0000 \\
\hline & 2,2 & 87,6014 & & Rejeita & & 0,0000 \\
\hline & 2,4 & 4,3185 & & Rejeita & & 0,0000 \\
\hline & 2,6 & 3,1186 & & Rejeita & & 0,0000 \\
\hline & 2,8 & 3,9401 & & Rejeita & & 0,0000 \\
\hline
\end{tabular}

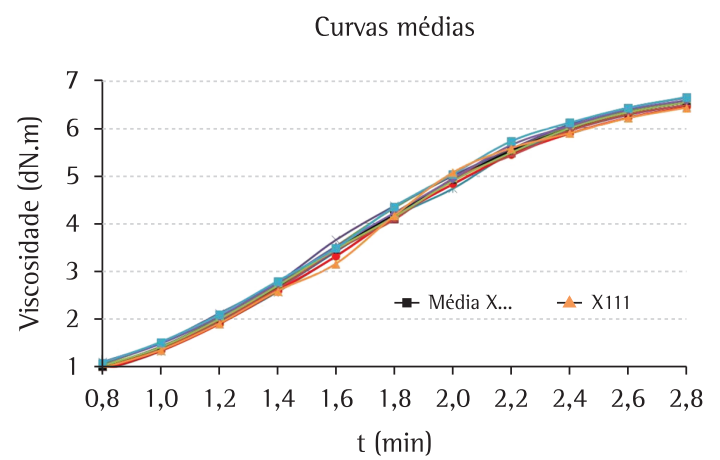

Figura 3. Curvas representativas do cenário simulado.

identificadas no gráfico (média geral e a curva do avaliador 1 sobre a peça 1 na primeira replicação). De $t=1,6$ a $t=2,2$ (aproximadamente $35 \%$ dos pontos) verifica-se variabilidade excessiva nas curvas. Essa variabilidade gera os resultados da ANOVA ponto a ponto que reprova o SM no intervalo. Como a ANOVA aprova o SM nos demais instantes, que são a maioria, uma decisão seria aprovar o SM. Essa conclusão é obtida na primeira abordagem, que utiliza a mediana, sendo assim mais conservadora. A segunda abordagem, baseada na média, aprova o SM porém colocando-o na região de aprovação condicional (entre 10\% e $30 \%)$. Esse exemplo deixa claros as características de cada abordagem e os problemas de decisão que podem decorrer do uso da ANOVA ponto a ponto.

A Tabela 13 apresenta os indicadores de capacidade e desempenho do SM no cenário de reprovação devida à VE, para as duas primeiras abordagens.

A Tabela 14 apresenta uma comparação entre os desvios na identidade da Equação 8 observados em cada cenário. 0 desvio mais próximo de zero ocorreu no cenário em que o SM foi aprovado; tal cenário foi gerado com os menores valores de erro aleatório, conforme pode-se verificar na Tabela 5. Os maiores desvios ocorreram para o cenário do SM reprovado
Tabela 13. Comparação dos percentuais de variação do SM para o cenário simulado.

\begin{tabular}{ccc}
\hline Fonte de variação & Mediana & Média \\
\hline \%VE & 2,3 & 21,8 \\
\%VA & 0,5 & 7,3 \\
\%VP & 100,0 & 97,3 \\
\%R\&R & 2,3 & 23,0 \\
$n d c$ & 60,21 & 5,98 \\
\hline
\end{tabular}

Tabela 14. Comparação dos desvios da identidade da Equação 8 por cenário simulado.

\begin{tabular}{lcccc}
\hline $\begin{array}{l}\text { Abordagem/ } \\
\text { cenário }\end{array}$ & $\begin{array}{c}\text { SM } \\
\text { aprovado }\end{array}$ & $\begin{array}{c}\text { SM rejeitado } \\
\text { (VA) }\end{array}$ & $\begin{array}{c}\text { SM rejeitado } \\
\text { (VE') }\end{array}$ & $\begin{array}{c}\text { SM rejeitado } \\
\text { (VE })\end{array}$ \\
\hline Mediana & 0,00000 & $-0,00061$ & 0,0541 & 0,00063 \\
Média & 0,00000 & $-0,00027$ & $-0,01220$ & $-0,00271$ \\
\hline
\end{tabular}

devido à VE', o qual foi gerado com a maior variação do erro aleatório. Esse resultado se justifica já que o aumento da variação do SM aumenta a probabilidade de diferentes valores esperados serem usados no cálculo da $S Q R$, definida pela Equação 30.0 desvio também aumenta quando se altera o método de cálculo da mediana para a média. Entretanto, no geral a magnitude do desvio não é suficiente para levar a uma conclusão errônea na ANOVA, conforme pode-se constatar analisando a ANOVA ponto a ponto em cada caso.

\section{Conclusão}

A avaliação do desempenho de produtos e processos industriais que envolvem a análise de dados funcionais configura-se como necessidade crescente na área da Engenharia da Qualidade. Dados funcionais ocorrem quando uma observação da variável de resposta de interesse não é dada por um valor individual, mas por uma coleção de dados que formam um perfil ou curva. Produtos e processos caracterizados por variáveis funcionais devem ser analisados através de métodos apropriados a esse tipo de variável. Uma análise apropriada deve considerar conjuntamente todos os pontos observados na curva.

0 método da ANOVA das distâncias proposto nesse artigo é uma adaptação do estudo tradicional de R\&R para contemplar o tratamento e análise de dados funcionais. 0 método apresenta apenas um resultado final, no qual estão considerados todos os pontos da curva. 0 principal elemento da proposta foi a utilização da distância de Hasudorff (DH) como medida de proximidade entre curvas. No contexto de uma ANOVA, a medida de proximidade das curvas está para o nível de variação do SM como a medida de dispersão está para uma variável simples. Um método detalhado por equações e tabelas foi desenvolvido 
para avaliar as componentes da variação de um SM em que os dados são curvas.

A ANOVA das distâncias soluciona os problemas causados pelo uso equivocado de métodos para variáveis simples em estudos de $R \& R$ nos quais a variável de resposta é funcional. A ANOVA das distâncias pode ser usada com facilidade em planilhas eletrônicas comuns, sem a necessidade de programas computacionais complexos (como comprovam as planilhas programadas para o estudo de caso na seção 4, disponíveis mediante solicitação).

Algumas questões relevantes que surgiram no decorrer dessa pesquisa podem ser apontadas como sugestões relacionadas ao desdobramento dos métodos apresentados: (i) a generalização da ANOVA das distâncias para projetos experimentais incluindo variáveis funcionais com mais de dois fatores; (ii) adaptação do método para uso em ensaios destrutivos; (iii) investigação da influência do número de pontos usados na análise das curvas sobre o cálculo das somas quadráticas da ANOVA; e (iv) investigar a aplicabilidade do método para diferentes formas e características das variáveis funcionais..

\section{Referências}

ABRAMOVICH, F.; ANGELINI, C. Testing in mixed-effects FANOVA models. Journal of Statistical Planning and Inference, v. 136, p. 4326-4348, 2006. http://dx.doi. org/10.1016/j.jspi.2005.06.002

AUTOMOTIVE INDUSTRY ACTION GROUP - AIAG. Measurement Systems Analysis. 3rd ed. Detroit: Chrysler Corporation; Ford Motor Company and General Motors Corporation, 2002.

BARRENTINE, L. B. Concepts for $R \& R$ studies. 2nd ed. Milwaukee: ASQ, 2003.

BURDICK, R. K.; BORROR, C. M.; MONTGOMERY, D. C. Design and analysis of gauge $R \& R$ studies: making decisions with confidence intervals in random and fixed ANOVA models. Philadelphia; Alexandria: ASA-SIAM Series on Statistics and Applied Probability, 2005.
BURDICK, R. K.; BORROR, C. M.; MONTGOMERY, D. C. A Review of Methods for Measurement Systems Capability Analysis. Journal of Quality Technology, v. 35, p. 342354, 2003.

CUEVAS, A.; FEBRERO, M.; FRAIMAN, R. An ANOVA test for functional data. Computacional Statistics \& Data Analysis, v. 47, p. 111-122, 2004. http://dx.doi. $\operatorname{org} / 10.1016 /$ j.csda.2003.10.021

FERRATY, F.; VIEU, P. Curves discrimination: a nonparametric functional approach. Computational Statistics \& Data Analysis v. 44, p. 161-173, 2003. http://dx.doi. org/10.1016/S0167-9473(03)00032-X

FOGLIATTO, F. S. Multiresponse optimization of products with functional quality characteristics. Quality and Reliability Engineering International, v. 24, n. 8, p. 927939, 2008. http://dx.doi.org/10.1002/qre.939

HUTTENLOCHER, D. P.; KLANDERMAN, G. A; RUCKLIDGE, W. J. Comparing images using the Hausdorff distance. IEEE Transactions on Pattern Analysis and Machine Intelligence, v. 15, p. 850-863, 1993. http://dx.doi. org/10.1109/34.232073

INTERNATIONAL VOCABULARY OF METROLOGY - VIM. Basic and general concepts and associated terms. 3rd ed. Joint Committee for Guides in Metrology, 2008. http:// dx.doi.org/10.1590/S0103-65132007000300002

KANG, L.; ALBIN, S. L. On-line monitoring when the process yields a linear profile. Journal of Quality Technology, v. 32, p. 418-426, 2000.

MAJESKE, K. D. Approval Criteria for Multivariate Measurement System. Journal of Quality Technology, v. 40, p. $140-153,2008$.

MONTGOMERY, D. C.; RUGER, G. C. Applied Statistics and Probability for Engineers. 3rd ed. New York: John Wiley and Sons, 2003.

MONTGOMERY, D. C. Design and Analysis of Experiments. New York: John Wiley and Sons, 2001.

RAMSAY, J. 0.; SILVERMAN, B. W. Functional data analysis. 2nd ed. New York: Springer, 2005.

SOUZA, C. P. E. Testes de hipóteses para dados funcionais baseados em distâncias: um estudo usando splines. 2008. Dissertação (Mestrado em Estatística)-Universidade Estadual de Campinas, Campinas, 2008.

WOODALL, W. H. Current research on profile monitoring. Produção, v. 17, p. 420-425, 2007.

\section{Repeatability and reproducibility studies of functional data}

\section{Abstract}

This paper presents a method to analyze the performance of measurement systems in a functional data analysis context, based on repeatability and reproducibility (R\&R) studies. Functional data are a collection of data points organized as a profile or curve. The method is an alternative to traditional methods that may lead to wrong conclusions when applied to functional data. In the proposed method, hypothesis tests and two-way ANOVA were adapted to be used in measurement system analysis. The method is grounded on the use of the Hausdorff distance to measure the proximity between curves. The method proposed was applied in a simulated repeatability and reproducibility study. The study was structured such that scenarios in which the measurement system was approved and rejected were tested. Results were compared with those obtained applying traditional ANOVA point wise on the curves. The method proposed was named ANOVA of the distances.

\section{Keywords}

Functional data analysis. Measurement systems. R\&R studies. ANOVA. Functional ANOVA. 